\title{
Wavelet Threshold-Based ECG Data Compression Technique Using Immune Optimization Algorithm
}

\author{
Mohammed Abo-Zahhad, Sabah M. Ahmed and Nabil Sabor \\ Electrical and Electronics Engineering Department, Faculty of Engineering, Assiut \\ University, Assiut, Egypt \\ (zahhad@yahoo.com,sabahma@yahoo.com,nabil.kolta@eng.au.edu.eg). \\ Ahmad F. Al-Ajlouni \\ Communication Engineering Department, Hijjawi Faculty for Engineering \\ Technology, Yarmouk University, Irbid, Jordan, (alajlouni@hotmail.com)
}

\begin{abstract}
In this paper, a new ECG compression method called Wavelet Threshold Based Immune Algorithm (WTBIA) is proposed. This method based on finding the best threshold level for each wavelet subband using Immune Algorithm (IA). The WTBIA algorithm consists of three main steps: 1) Applying 1-D Discrete Wavelet Transform (DWT) on ECG signal; 2) Thresholding of wavelet coefficients in each subband; and 3) Minimization of the Percent Root mean square Difference (PRD) and maximization of the Compression Ratio (CR) using IA. The main advantage of this method is finding the best threshold level for each subband based on the required $C R$ and PRD. The compression algorithm was implemented and tested upon records selected from the MIT-BIH arrhythmia database [6] using different wavelets such as Haar, Daubechies, Coiflet, Symlet and Biorthogonal. Simulation results show that the proposed algorithm leads to high CR associated with low distortion level relative to previously reported compression algorithms.
\end{abstract}

Keywords: Electrocardiogram, Data Compression, Wavelet Transform, Immune Algorithm

\section{Introduction}

An Electrocardiogram (ECG) is one of the most important physiological signals with essential information for heart disease diagnosis. The ECGs are almost periodic, consisting of cardiac beat pulses with periods of approximately one second [7]. ECG compression has high importance to reduce storage requirements and/or the transmission rate for ECG data storage and archiving, ambulatory recording systems and ECG data transmission over telephone line or digital telecommunication network. The desired objective is to provide a high-quality reconstruction of electrocardiogram signals at low bit rates and acceptable distortion levels [1]. Most compression algorithms belong to the following categories [7]: 1) Direct data compression methods, which detect redundancies by analysing the signal samples; 2) Pattern recognition methods, in which some parameters of the signal are extracted and used for compression; 3) Predictive methods, which calculate the error between the real and the predicted data, then quantize and code both the error and the information needed to reproduce the prediction; or 4) Transform methods, which firstly transform the signal into another time-frequency representation better suited for detecting and removing redundancies. 
Wavelet Threshold Based Immune Algorithm (WTBIA) proposed in this paper belongs to the latter category. The goal of most modern wavelet research is to create a set of basis functions that provides an efficient and useful description of a signal. The wavelet transform has been successful in providing high compression ratios while maintaining good signal quality [1-2]. The efficiency of ECG compression using wavelets depends on the shape of the wavelet [7]; so in this paper the ECG signal is decomposed by using the discrete wavelet transform up to the fifth level using different wavelets such as Haar, Daubechies, Coiflet, Symlet and Biorthogonal. The paper is organized as follows. Section 2 is literature review. Section 3 describes the proposed method in detail and how the threshold level is obtained using immune algorithm. In Section 3 the algorithm is tested on selected records from the MIT-BIH arrhythmia database and compared with other method in the literature in [11-16]. Finally, Section 4 offers some conclusions.

\section{Related Work}

In few past years, many compression algorithms have been adopted for compression of ECG signal [11-16]. A wavelet-based electrocardiogram (ECG) data compression algorithm is adopted in [11]. The ECG signal is first preprocessed, the discrete wavelet transform (DWT) is then applied to the preprocessed signal. Preprocessing guarantees that the magnitudes of the wavelet coefficients be less than one, and reduces the reconstruction errors near both ends of the compressed signal. The DWT coefficients are divided into three groups, each group is thresholded using a threshold based on a desired energy packing efficiency. A binary significance map is then generated by scanning the wavelet decomposition coefficients and outputting a binary one if the scanned coefficient is significant and a binary zero if it is insignificant. In [12] a wavelet ECG data codec based on the Set Partitioning in Hierarchical Trees (SPIHT) compression algorithm has been developed. Authors modified the SPIHT algorithm for the one-dimensional case and applied it to compression of ECG data.

An ECG compression algorithm, called Analysis by Synthesis ECG compressor (ASEC), has been introduced in [13]. The ASEC algorithm is based on analysis by synthesis coding, and consists of a beat codebook, long and short-term predictors, and an adaptive residual quantizer. The compression algorithm uses a defined distortion measure in order to efficiently encode every heartbeat, with minimum bit rate, while maintaining a predetermined distortion level. The compression algorithm was implemented and tested with both the percentage rms difference (PRD) measure and the recently introduced weighted diagnostic distortion (WDD) measure. In [14], an ECG signal is compressed based DWT and QRS-complex estimation. The ECG signal is preprocessed by normalization and mean removal. Then, an error signal is formed as the difference between the preprocessed ECG signal and the estimated QRS-complex waveform. This error signal is wavelet transformed and the resulting wavelet coefficients are threshold by setting to zero all coefficients that are smaller than certain threshold levels. The threshold levels of all subbands are calculated based on Energy Packing Efficiency (EPE) such that minimum percentage root mean square difference (PRD) and maximum compression ratio (CR) are obtained. Authors in [15] developed an ECG compression algorithm based on the use of wavelet transform, leading to low/high frequency components separation, high order statistics based thresholding, using level adjusted kurtosis value, to de-noise the ECG signal, and next a linear predictive coding filter is applied to the wavelet coefficients producing a lower variance signal. This latter one will be coded using the Huffman encoding yielding an optimal 
coding length in terms of average value of bits per sample. At the receiver end point, with the assumption of an ideal communication channel, the inverse processes are carried out namely the Huffman decoding, inverse linear predictive coding filter and inverse discrete wavelet transform leading to the estimated version of the ECG signal.

In [16], authors presented a new algorithm for the segmentation of ECG signal into waves and segments for compression application. The developed compression algorithm begins by segmenting the ECG signal into its main components (P-waves, QRScomplexes, T-waves, U-waves and the isoelectric waves). The resulting waves are grouped into Region of Interest (RoI) and Non Region of Interest (NonRoI) parts. Consequently, lossless and lossy compression schemes are applied to the RoI and NonRoI parts respectively. Ideally we would like to compress the signal losslessly, but in many applications this is not an option. Thus, given a fixed bit budget, it makes sense to spend more bits to represent those parts of the signal that belong to a specific RoI and, thus, reconstruct them with higher fidelity, while allowing other parts to suffer larger distortion. Most of previous compression algorithms used predefined threshold level, so a new ECG compression method called Wavelet Threshold Based Immune Algorithm (WTBIA) is proposed here. Immune Algorithm (IA) is one of recently optimization algorithms which that mimic the antigen-antibody reaction of the immune system in mammals. The antigen and the antibody in the IA are equivalent to the objective function and the feasible solution for a conventional optimization method. In the proposed compression method, IA is used to find the threshold level of each wavelet subband subject to minimization of the percent root mean square difference (PRD) and maximization of the compression ratio (CR).

\section{The Proposed WTBIA Method}

The WTBIA method is based on integrating the wavelet transform and the immune algorithm for finding the best threshold level that gives low PRD and high CR. The flow chart of the WTBIA method is shown in Figure (1). The algorithm is divided into three main steps: 1) Applying DWT; 2) Thresholding process; and 3) Using IA for finding the best threshold level.

\subsection{Discrete Wavelet Transform}

Wavelet transform is a powerful tool for digital signal processing. It has fine frequency resolution and coarse time resolution at lower frequency, and coarse frequency resolution and fine time resolution at higher frequency. The continuous wavelet transform (CWT) maps a one dimensional signal to a highly redundant joint time-scale representation. Discrete wavelet transform (DWT) removes the redundancy of CWT by using discrete steps for scale and translation. Figure 2-a shows an implementation of a three-level forward DWT based on a two-channel recursive filter bank, where $h_{0}(n)$ and $h_{1}(n)$ are low-pass and high-pass analysis filters respectively, and the block $\downarrow 2$ represents the down sampling operator by a factor of 2 . The input signal $x(n)$ is recursively decomposed into a total of four subband signals: a coarse signal $C_{3}(n)$, and three detail signals, $D_{3}(n), D_{2}(n)$ and $D_{1}(n)$, of three resolutions. 
International Journal of Signal Processing, Image Processing and Pattern Recognition Vol. 8, No. 2 (2015)

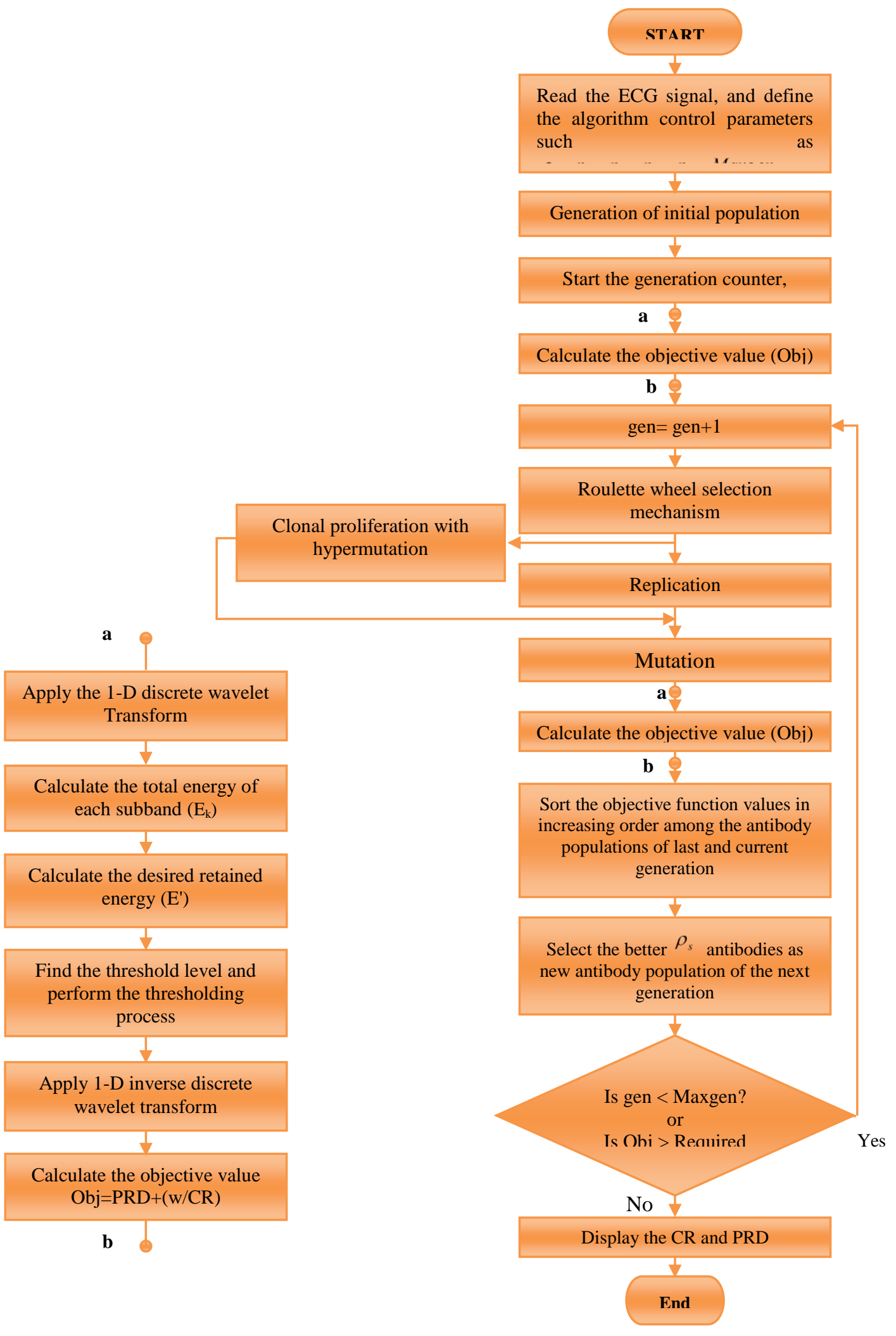

Figure 1. Flow Chart of WTBIA Proposed Method 
Figure 2-b shows an implementation of a three-level inverse DWT based on a twochannel recursive filter bank, where $\tilde{h}_{0}(n)$ and $\tilde{h}_{1}(n)$ are low-pass and high-pass synthesis filters respectively, and the block $\uparrow 2$ represents the up sampling operator by a factor of 2. The four subband signals $C_{3}(n), D_{3}(n), D_{2}(n)$ and $D_{1}(n)$, are recursively combined to reconstruct the output signal $\tilde{x}(n)$. The four finite impulse response filters satisfy the relationships given by Equations (1-3), so that the output of the inverse DWT is identical to the input of the forward DWT. In the proposed method, the ECG signal is decomposed by using the DWT up to the fifth level using different wavelets such as Haar, Daubechies, Coiflet, Symlet and Biorthogonal.

$$
\begin{aligned}
& h_{1}(n)=(-1)^{n} h_{0}(n) \\
& \tilde{h}_{0}(n)=h_{0}(1-n) \\
& \tilde{h}_{1}(n)=(-1)^{n} \tilde{h}_{0}(n)
\end{aligned}
$$

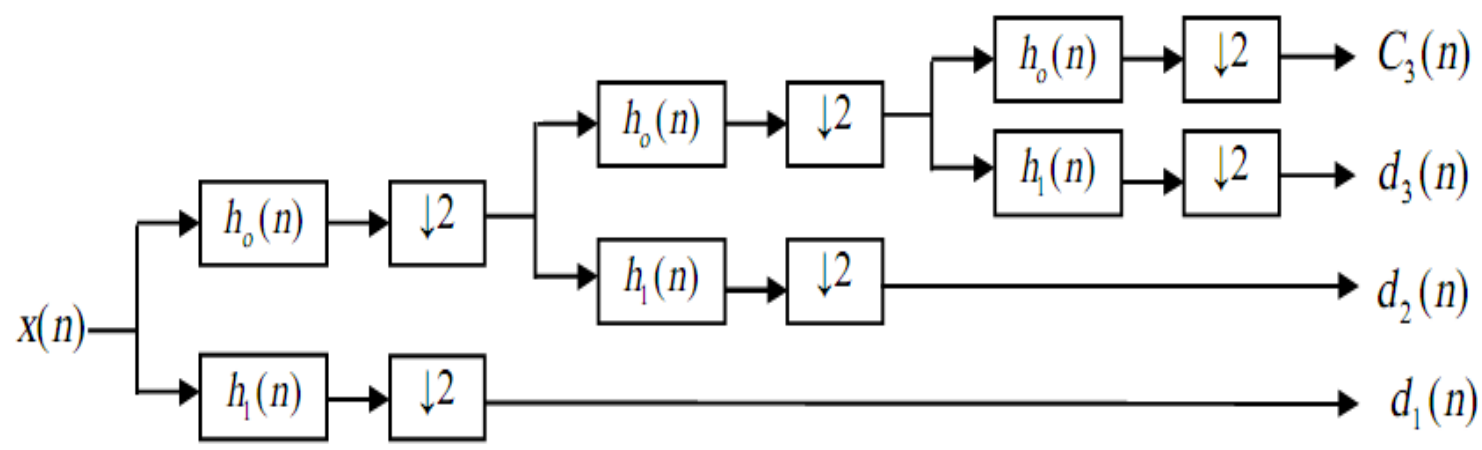

(a) Forward DWT

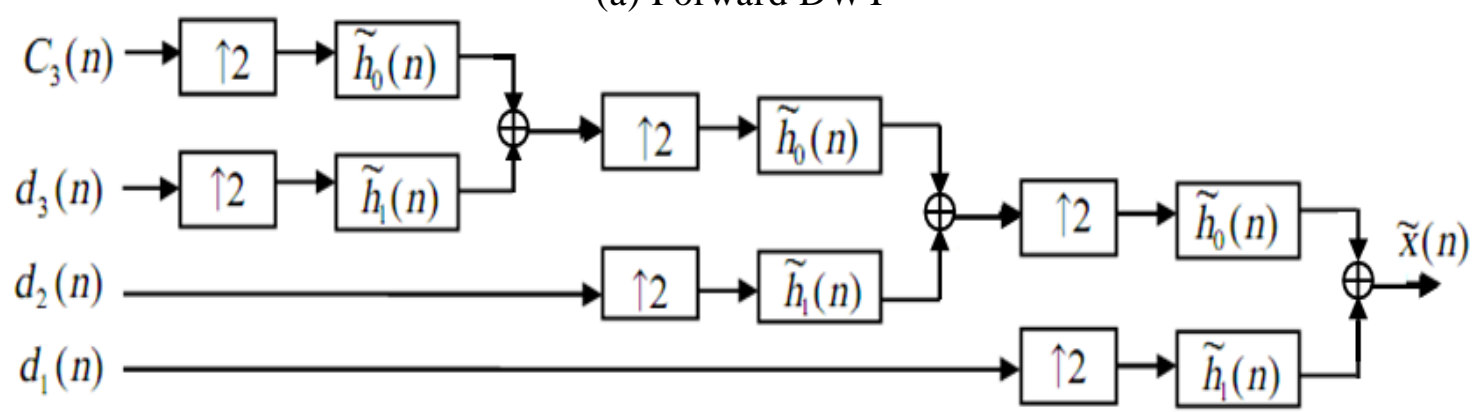

(b) Inverse DWT

Figure 2. 1-D Discrete Wavelets Transform

\subsection{Thresholding Process}

The main goal in compression is eliminated redundancy between samples of ECG signals, without any significant distortions in reconstructed signal. For this reason, in this paper the threshold level of each subband is determined using IA based on maximizing the CR and minimizing the PRD. The following steps show how the threshold level will be determined:

1. Calculate the total energy of each subband using the following equation:

$$
E_{k}=\sum_{k} y_{k}^{2}(i)
$$


where, $E_{k}$ and $y_{k}$ are the energy and the coefficients values of $\mathrm{k}^{\text {th }}$ subband, respectively.

2. Calculate the desired retained energy $E^{\prime}$ using the following equation:

$$
E^{\prime}=\sum_{j=1}^{N+1} \alpha_{k} E_{k}
$$

where $\alpha_{k}$ are positive numbers in range [0 1 l] and $\mathrm{N}$ is the number of wavelet levels.

3 . Form the sequence $F(i)$ by sorting the magnitudes of the wavelet coefficients in descending order.

4. Find the value of $\mathrm{L}$ under the following condition:

$$
\sum_{i=1}^{L} F^{2}(i) \geq E^{\prime}
$$

5. Then the threshold level is $\mathrm{F}(\mathrm{L})$.

6. Calculate the PRD and CR using the following equations:

$$
P R D=\sqrt{\frac{\sum_{i=1}^{n}(x(i)-\hat{x}(i))^{2}}{\sum_{i=1}^{n}(x(i))^{2}}} \times 100 \text { and } C R=\frac{\text { Number of bits of } y}{\text { Number of bits of } \hat{y}}
$$

where, $x$ and $\hat{x}$ are the original signal and the reconstruction signal respectively; $y$ and $\hat{y}$ are values of subband coefficients before and after thresholding process respectively.

7. The optimal values of $\alpha_{k}(k=1,2, \ldots, N+1)$ are obtained based on minimizing the following objective function:

$$
O b j=P R D+\frac{1}{C R}
$$

However, since the value of $1 / \mathrm{CR}$ is small relatively to the value of $\mathrm{PRD}$, a weighting factor $\mathrm{W}$ is introduced to increase the percentage of sharing of $1 / \mathrm{CR}$. The value of $\mathrm{W}$ is based on which is more important: high CR or low PRD. So, Equation (8) is rewritten in the form:

$$
O b j=P R D+\frac{W}{C R}
$$

\subsection{Immune Algorithm}

Immune algorithms are randomized algorithms inspired by immune functions and principles observed in nature [3-5]. Such algorithms begin by generating population pool (chromosomes) using real coding representation and evaluating the objective values. Then, the population pool undergoes the algorithm operations which will be described in this section. The operations are repeated at each generation (gen) until the termination condition is satisfied. Table 1 illustrates the main steps of the immune algorithm. 


\section{Table 1. Steps of Immune Algorithm}

\begin{tabular}{|c|c|}
\hline gen $=1$; & $\%$ The first generation \\
\hline Chrom=Initial_pop () ; & $\%$ Construct the initial population pool \\
\hline \multicolumn{2}{|l|}{ While (termination_condition is false) } \\
\hline Evaluate (Chrom); & $\%$ Evaluation of initial population \\
\hline Chrom_sel=RWS_Selection(Chrom); & $\%$ Roulette wheel selection \\
\hline Chrom_rep=replication(Chrom_sel); & $\begin{array}{l}\% \text { Selection of better antibodies using } \\
\text { Replication }\end{array}$ \\
\hline Chrom_clon=Cloning $($ Chrom_rep $)$; & $\%$ Clonal operation \\
\hline Chrom_hyper=Hypermutation(Chrom_clon) & \% Hypermutation operation \\
\hline Chrom_tot=[ Chrom_rep, Chrom_hyper $]$; & $\%$ Mutation Operation \\
\hline Chrom_child=Mutation(Chrom_tot); & \% Objective function evaluation \\
\hline Evaluate (Chrom_child); & $\%$ Selection of better antibodies for next \\
\hline Chrom=Better_selection(Chrom,Chrom_chil & generation \\
\hline d) & $\%$ Increment the number of generations \\
\hline \multicolumn{2}{|l|}{ gen $=$ gen +1} \\
\hline end & \\
\hline
\end{tabular}

\section{A. Generation of Antibody Population}

The antibody population is randomly generated with size $\rho_{s}$ using real coding representation. Each antibody is encoded as a vector of floating point numbers, with the same length as the vector of decision variables. For $N$ levels wavelet, there are $(N+1)$ decision variables in each antibody (solution) $Q_{j}=\left(\alpha_{1}, \alpha_{2}, \ldots, \alpha_{I}, \alpha_{J}, \alpha_{K}, \ldots, \alpha_{N+1}\right), j=1,2, \ldots, \rho_{s}$.

\section{B. Selection for Reproduction}

The roulette wheel selection is employed in immune bases algorithms for chromosomes reproduction. The basic idea of the roulette wheel is to select some solutions randomly from population pool based on probability of each solution.

\section{Replication Operation}

The replication operation is used to select better solutions, which have low objective values to undergo algorithm operations. This is termed by clonal proliferation within hypermutation and mutation operations.

\section{Clonal Proliferation within Hypermutation}

Based on the biological immune principles, the selection of a certain antibody from the antibody population pool to join the clonal proliferation depends on the clonal selection rate $\left(p_{c}\right)$. Each gene (coefficient), in a single antibody, depending on the hypermutation rate $\left(p_{h}\right)$, executes the hypermutation of convex combination. The hypermutation rate $\left(p_{h}\right)$ has an extremely high rate than the mutation rate to increase the 
antibody diversity. For a given solution $Q_{j}=\left(\alpha_{1}, \alpha_{2}, \ldots, \alpha_{I}, \alpha_{J}, \alpha_{K}, \ldots, \alpha_{N+1}\right)$, if the coefficient $\alpha_{I}$ is determined to execute the hypermutation and another coefficient $\alpha_{K}$ is randomly selected to join in; the resulting offspring solution becomes $Q_{j}^{\prime}=\left(\alpha_{1}, \alpha_{2}, \ldots, \alpha_{I}^{\prime}, \alpha_{J}, \alpha_{K}, \ldots, \alpha_{N+1}\right) \quad$, where the new coefficient $\alpha_{I}^{\prime}$ is $\alpha_{I}^{\prime}=(1-\beta) \alpha_{I}+\beta \alpha_{K}$, and $\beta \in[0,1]$ is a random value.

\section{E. Mutation Operation}

Similar to the hypermutation mechanism, the mutation operation is also derived from the convex set theory, where each coefficient, in a single solution, depending on the mutation rate $\left(p_{m}\right)$, executes the mutation of convex combination. Two coefficients in a single solution are randomly chosen to execute the mutation of convex combination. For a given solution $Q_{j}=\left(\alpha_{1}, \alpha_{2}, \ldots, \alpha_{I}, \alpha_{J}, \alpha_{K}, \ldots, \alpha_{N+1}\right)$, if the coefficient $\alpha_{I}$ is determined to execute the mutation depend on the mutation rate $(\mathrm{pm})$ and another coefficient $\alpha_{K}$ is randomly selected to join in; the resulting offspring is $Q_{j}^{\prime}=\left(\alpha_{1}, \alpha_{2}, \ldots, \alpha_{I}^{\prime}, \alpha_{J}, \alpha_{K}^{\prime}, \ldots, \alpha_{N+1}\right)$. The resulting new two coefficients $\alpha_{I}^{\prime}$ and $\alpha_{K}^{\prime}$ are calculated as:

$$
\alpha_{I}^{\prime}=(1-\beta) \alpha_{I}+\beta \alpha_{K} \text { and } \alpha_{K}^{\prime}=\beta \alpha_{I}+(1-\beta) \alpha_{K}
$$

where, $\beta$ is selected randomly in the range $[0,1]$.

\section{F. Selection Operation}

The selection operation is generally used to select the better $\rho_{s}$ solutions which have low objective values as the new antibody population of the next generation.

\section{G. Stopping Criterion}

The stopping criterion achieves when the objective function doesn't change for certain number of generations or when the number of generations exceeds the specified maximum generations (Maxgen).

\section{Experiments Results and Discussion}

In order to study the effect of different wavelets (such as Haar, Daubechies, Coiflet, Symlet and Biorthogonal) on efficiency of the proposed method, the records number 117 and 208 consisting of the first 2048 and 1024 samples respectively from the MIT BIH Arrhythmia database have been used [6]. The ECG signals were digitized through sampling at $360 \mathrm{~Hz}$, quantized and encoded with 11 bits.

Table 2 shows the CR and PRD of two records 117 and 208 for Haar, Daubechies, Coiflet, Symlet and Biorthogonal wavelets. Figure 3 and Figure 4 show the original, reconstructed and reconstruction error signals of ECG record no. 117 for Biorthogonal 4.4 and Haar wavelets, respectively. The original, reconstructed and reconstruction error signals of ECG record no. 208 for Biorthogonal 4.4 and Haar wavelets, respectively are shown in Figure 5 and Figure 6. From these Figures and Table 2, it can be noticed that the best results from the PRD point of view were obtained using the Biorthogonal 4.4 wavelet due to its similarity with the shape of the QRS complex and the worst results obtained using the Haar wavelet due to discontinuities of the Haar wavelet. 
In order to compare the performance of the proposed method with other methods described in [11-14], the records number 101, 117, 119, 210 and 207 consisting of the first 2048 samples from the MIT-BIH Arrhythmia database have been used [6]. From Table 3 it can be seen that the results of the proposed method for different records are better than the results obtain with other methods [11-14].

\section{Table 2. The PRD and CR Values of the Two Records 117 and 208 at Various Wavelets}

\begin{tabular}{|c|c|c|c|c|}
\hline \multirow{2}{*}{ Wavelet function } & \multicolumn{2}{|c|}{ Record No. 117 } & \multicolumn{2}{c|}{ Record No. 208 } \\
\cline { 2 - 5 } & CR & PRD \% & CR & PRD \% \\
\hline Haar & 13.26 & 0.8609 & 11.3 & 1.2190 \\
\hline Coiflet 4 & 13.26 & 0.4435 & 11.3 & 0.7547 \\
\hline Daubechies 4 & 13.26 & 0.4313 & 11.3 & 0.6188 \\
\hline Symlet 4 & 13.26 & 0.4161 & 11.3 & 0.5754 \\
\hline Biorthogonal 4.4 & 13.26 & 0.3965 & 11.3 & 0.5217 \\
\hline
\end{tabular}

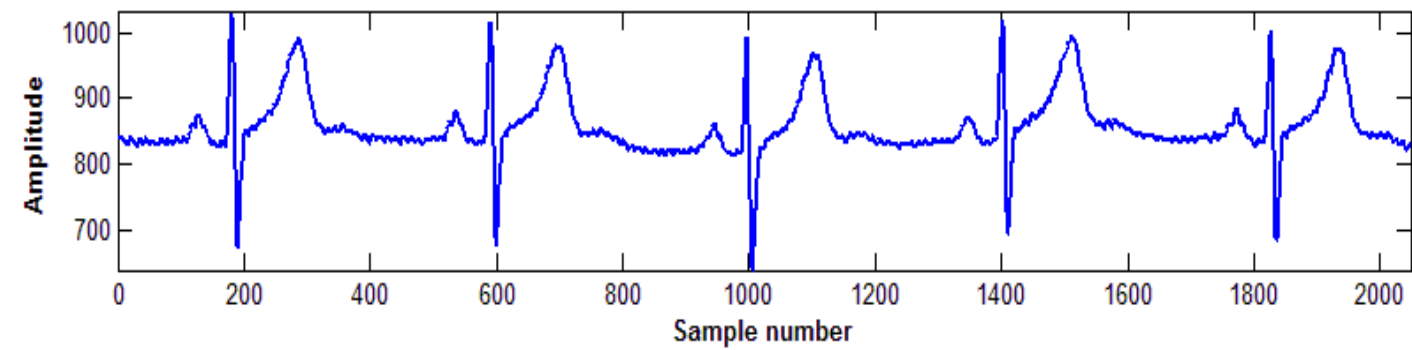

(a) Original signal

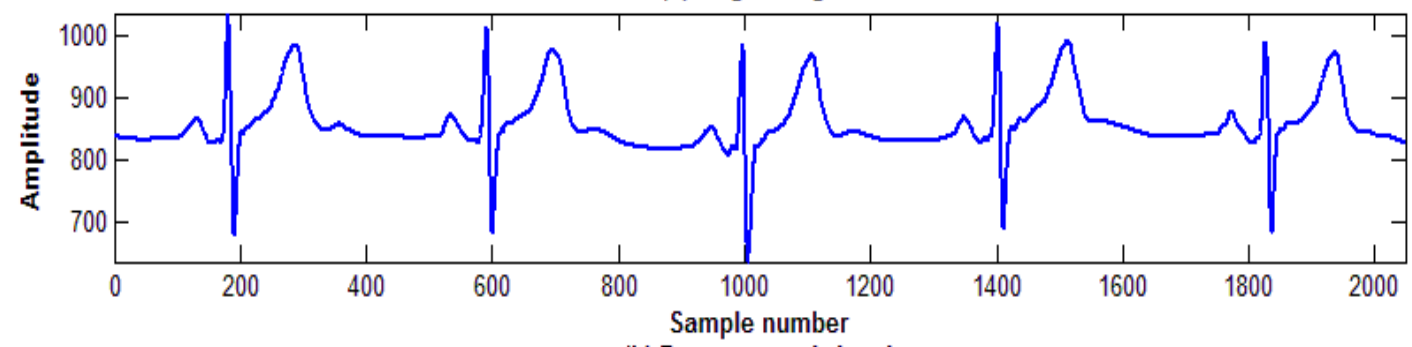

(b) Reconstructed signal

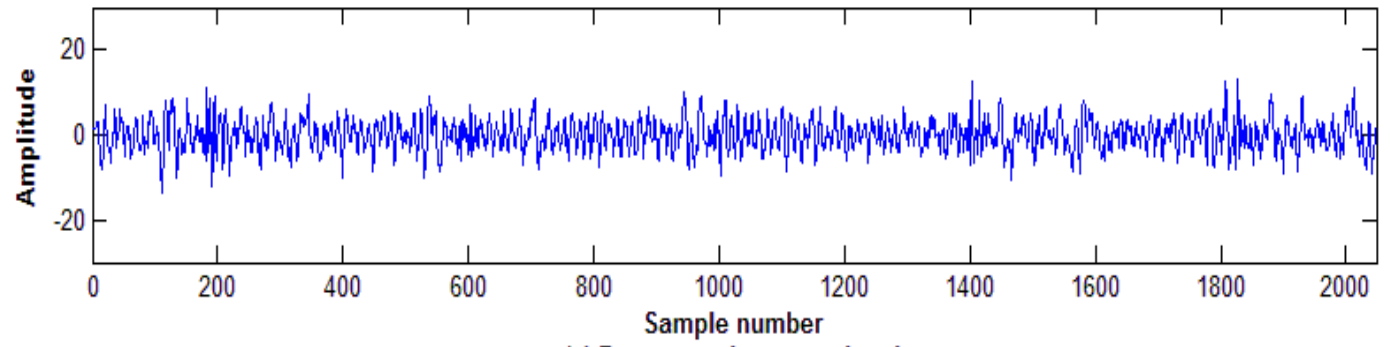

(c) Reconstruction error signal

Figure 3. The Original, Reconstructed and Error of ECG Record no. 117 for Bior 4.4 Wavelet 


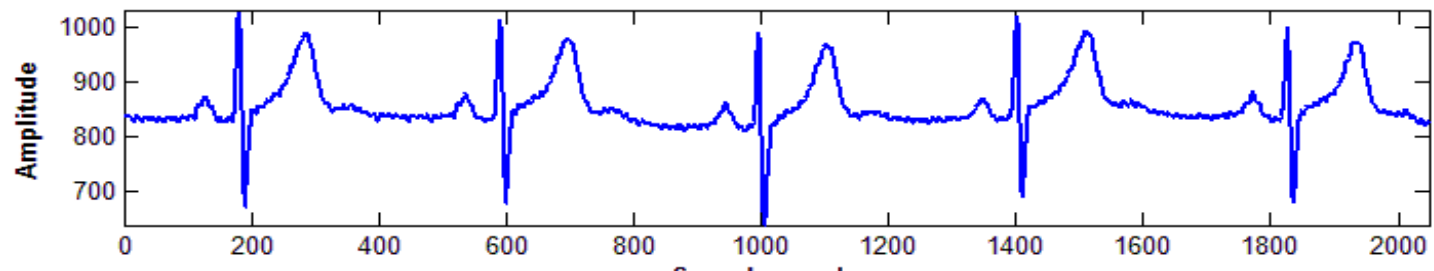

(a) Original signal

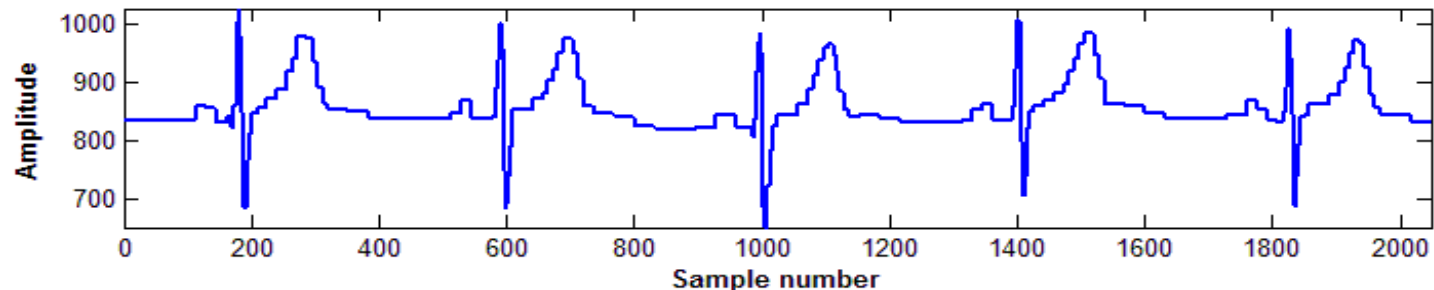

(b) Reconstructed signal

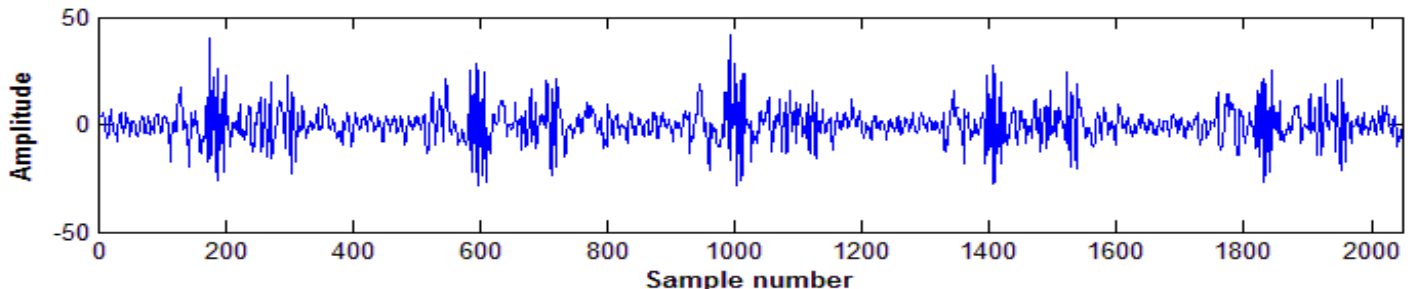

(c) Reconstruction error signal

Figure 4. The Original, Reconstructed and Error of ECG Record no. 117 for Haar Wavelet
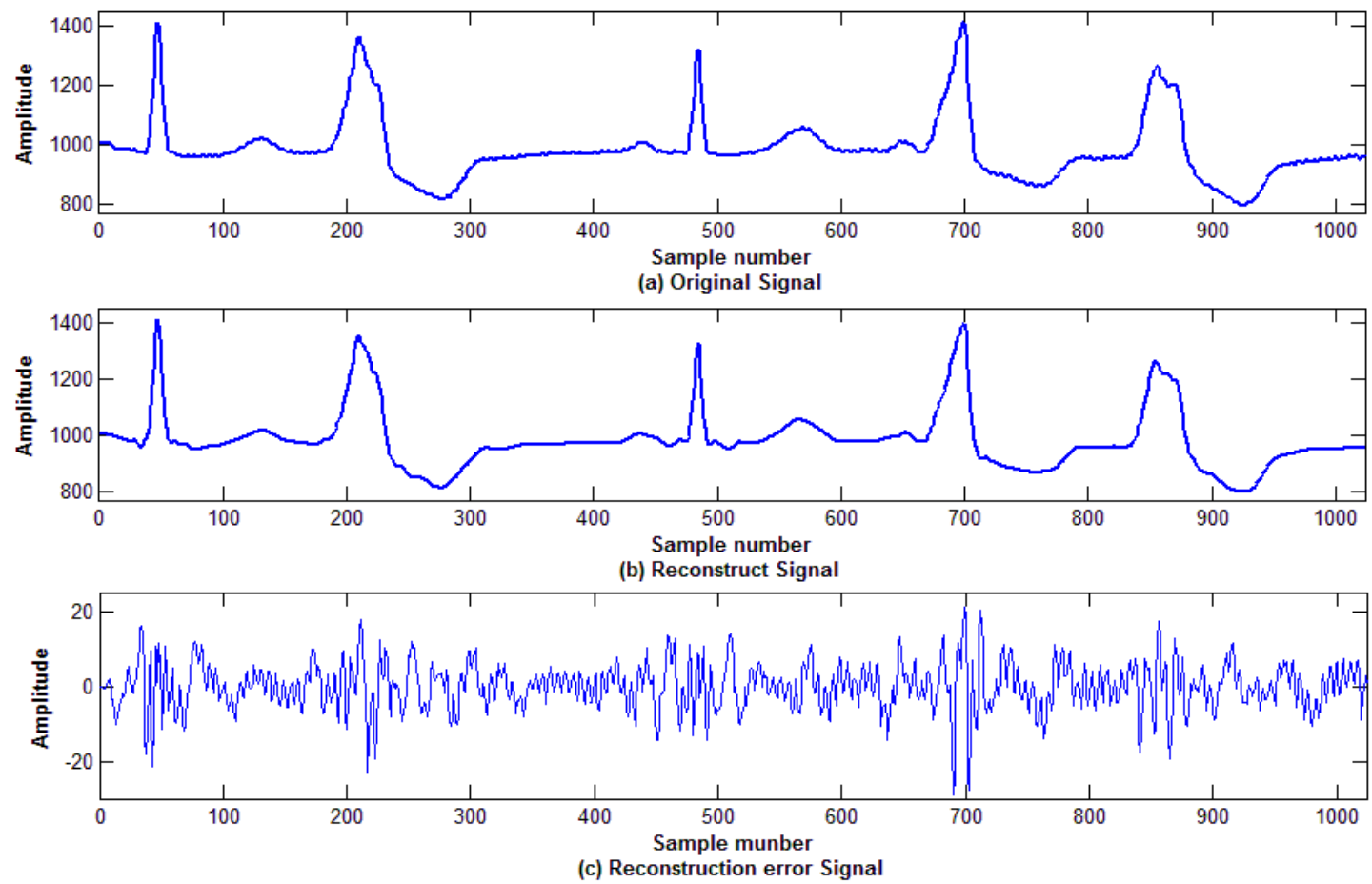

Figure 5. The Original, Reconstructed and Error of ECG Record no. 208 for Bior 4.4 Wavelet 

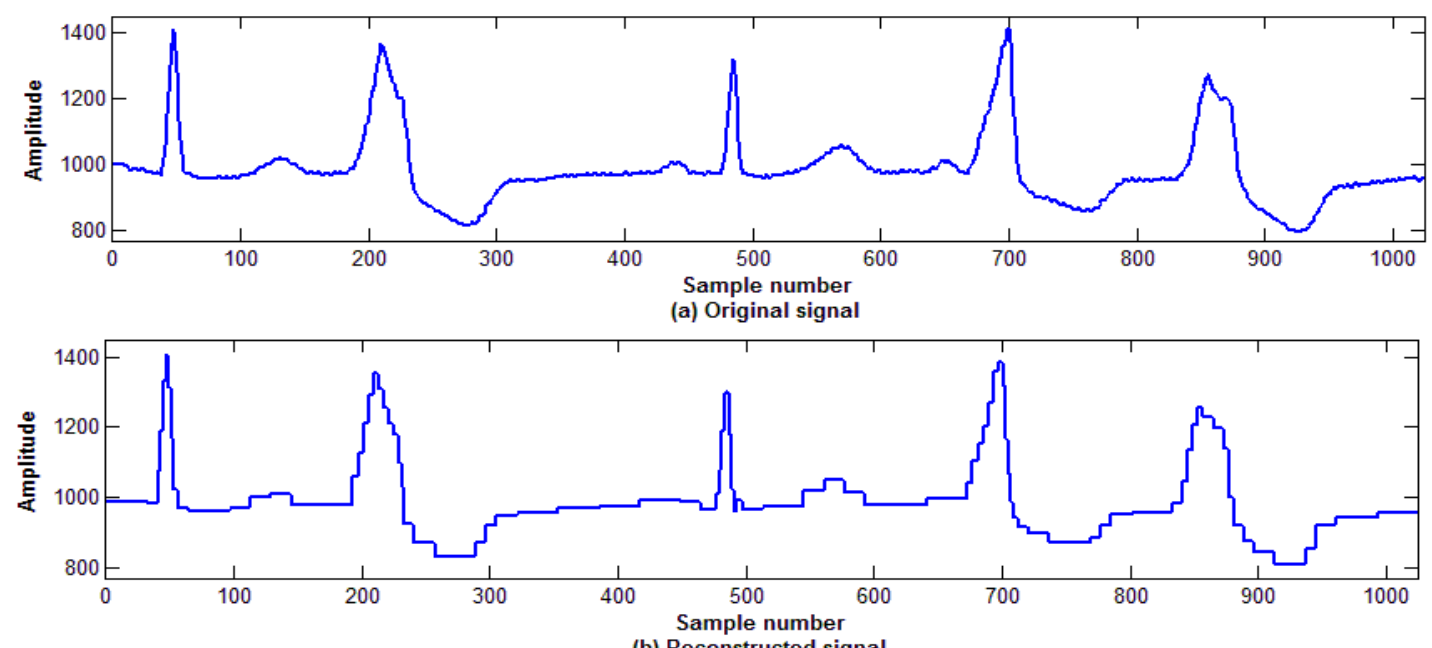

(b) Reconstructed signal

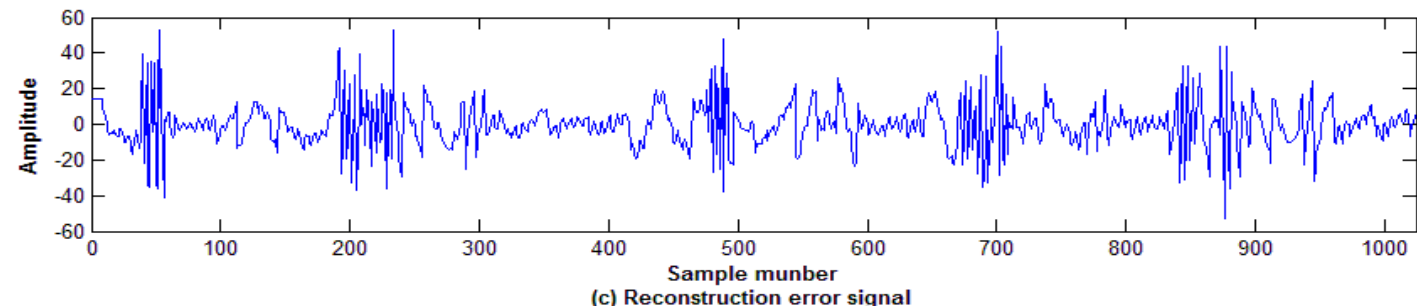

Figure 6. The Original, Reconstructed and Error of ECG Record no. 208 for Haar Wavelet

Table 3. Comparison between the Proposed Compression Method and Other Compression Methods

\begin{tabular}{|c|c|c|c|}
\hline Record & Method & CR & PRD \% \\
\hline \multirow{4}{*}{101} & Method described in [13] & 26.64 & 9.14 \\
\cline { 2 - 4 } & Method described in [14] & 26.70 & 1.77 \\
\cline { 2 - 4 } & Proposed method & $\mathbf{2 6 . 8 8}$ & $\mathbf{1 . 3 8}$ \\
\hline \multirow{4}{*}{117} & Method described in [11] & 10.80 & 0.48 \\
\cline { 2 - 4 } & & 22.19 & 1.06 \\
\cline { 2 - 4 } & Method described in [12] & 8 & 1.18 \\
\cline { 2 - 4 } & Method described in [15] & 14 & 4.75 \\
\cline { 2 - 4 } & Method described in [16] & 15.6 & 2.81 \\
\cline { 2 - 4 } & Proposed method & $\mathbf{1 3 . 2 6}$ & $\mathbf{0 . 3 9}$ \\
\cline { 2 - 4 } & & $\mathbf{2 5 . 2 7}$ & $\mathbf{0 . 9 7}$ \\
\hline \multirow{5}{*}{$\mathbf{1 1 9}$} & Method described in [11] & 23 & 1.95 \\
\cline { 2 - 4 } & Method described in [14] & 23 & 1.95 \\
\cline { 2 - 4 } & Proposed method & $\mathbf{2 4 . 6 7}$ & $\mathbf{1 . 5 6}$ \\
\hline \multirow{5}{*}{210} & Method described in [11] & 11.55 & 0.44 \\
\cline { 2 - 4 } & Method described in [14] & 11.55 & 0.49 \\
\cline { 2 - 4 } & Proposed method & $\mathbf{1 2 . 4 1}$ & $\mathbf{0 . 3 2}$ \\
\hline \multirow{5}{*}{207} & Method described in [11] & 23 & 0.55 \\
\cline { 2 - 4 } & Proposed method & $\mathbf{2 3}$ & $\mathbf{0 . 4 7}$ \\
\hline
\end{tabular}




\section{Conclusions}

In this paper, a new ECG compression method based on wavelet transform and immune algorithm called WTBIA has been proposed. This method uses immune algorithm to find the best threshold level for each wavelet subband based on the required CR and PRD. The WTBIA algorithm consists of three main steps: 1) Applying 1-D discrete wavelet transform on ECG signal; 2) Thresholding of wavelet coefficients in each subband; and 3) Finding the best threshold level based on minimization of the percent root mean square difference and maximization of the compression ratio using immune algorithm. The main advantage of this method is that the threshold level for each subband isn't constant for all wavelet subbands but it is determined for each subband using immune algorithm based on the required CR and PRD. The compression algorithm was implemented and tested upon records selected from the MIT-BIH arrhythmia database [6] using different wavelets such as Haar, Daubechies, Coiflet, Symlet and Biorthogonal. Simulation results show that the proposed method leads to high CR associated with low distortion level relative to previously reported compression algorithms [11-16].

\section{References}

[1] T. I. Mohammadpour and M. R. K. Mollaei, "ECG Compression with Thresholding of 2-D Wavelet Transform Coefficients and Run Length Coding", European Journal of Scientific Research, vol. 2, no. 27, (2009).

[2] O. O. Khalifa, S. H. Harding and A. A. Hashim, "Compression Using Wavelet Transform" Signal Processing: An International Journal (SPIJ), vol. 5, no. 2, (2008).

[3] M. Abo-Zahhad, S. M. Ahmed, N. Sabor and A. F. Al-Ajlouni, "Design of Immune Algorithm Based TwoDimensional Recursive Digital Filters Using Multi-level Orthogonal Arrays", IEEE $28^{\text {th }}$ National Radio Science Conference (NRSC2011), (2011) April 26-28, Cairo.

[4] M. Abo-Zahhad, S. M. Ahmed, N. Sabor and A. F. Al-Ajlouni, "Design of Two-Dimensional Recursive Digital Filters with Specified Magnitude and Group Delay Characteristics using Taguchi-based Immune Algorithm", Int. J. of Signal and Imaging Systems Engineering, vol. 3, no. 3, (2010).

[5] M. Abo-Zahhad, S. M. Ahmed, N. Sabor and A. F. Al-Ajlouni, " The Convergence Speed of Single-and Multi-Objective Immune Algorithm Based Optimization Problems", Signal Processing: An International Journal, vol. 5, no. 4, (2010).

[6] "MIT-BIH Arrhythmia Database", www.physionet.org/physiobank/database/mitdb.

[7] S. R. do Sapucai, C. M. Agulhari, I. S. Bonatti and P. L. D. Peres," A Genetic Algorithm to Compress Electrocardiograms Using Parameterized Wavelets", IEEE International Symposium on Signal Processing and Information Technology, (2007) December, Giza.

[8] Y. Takehisa, H. Sakanashi and T. Higuchi, "Adaptive Wavelet Transform for Lossless Compression Using Genetic Algorithm", Genetic and Evolutionary Computation Conference, (2000) July.

[9] E. Jones, P. Runkle, N. Dasgupta and L. Carin, "Signal Adaptive Wavelet Design Using Genetic Algorithms", IEEE Transactions on Pattern Analysis and Machine Intelligence, vol. 23, no. 23, (2001).

[10] M. A. F. Al-Husainy, "Sound Data Compression Method Using Genetic Algorithm", Damascus Univ. Journal, vol. 2, no. 23, (2007).

[11] B. A. Rajoub, "An Efficient Coding Algorithm for the Compression of ECG Signals Using the Wavelet Transform”, IEEE Transactions on Biomedical Engineering, vol. 4, no. 49, (2002).

[12] Z. Lu, D. Y. Kim and W. A. Pearlman, "Wavelet compression of ECG signals by the set partitioning in hierarchical trees algorithm," IEEE Trans. on Biomedical Engineering, vol. 7, no. 47, (2000).

[13] Y. Zigel, A. Cohen and A. Katz," ECG Signal Compression Using Analysis by Synthesis Coding”, IEEE Transactions on Biomedical Engineering, vol. 10, no. 47, (2000).

[14] M. Abo-Zahhad, S. M. Ahmed and A. Zakaria, "ECG Signal Compression Technique Based on Discrete Wavelet Transform and QRS-Complex Estimation", Signal Processing-An International Journal (SPIJ), vol. 2, no. 4, (2011).

[15] S. A. Chouakri, O. Djaafri and A. Taleb-Ahmed, "Wavelet Transform and Huffman Coding Based Electrocardiogram Compression Algorithm: Application to Telecardiology", $24^{\text {th }}$ IUPAP Conf. on Computational Physics, (2012) October 14-18, Kobe, Japan. 
[16] T. K. Abdel-Hamid, M. M. Abo-Zahhad and A. M. Mohamed," A New ECG Signal Compression Algorithm Based on Signal Segmentation into Waves and Segments", IEEE $31^{\text {st }}$ National Radio Science Conference (NRSC2014), Faculty of Engineering, (2014) April 28-30, Ain Shams University, Egypt.

\section{Authors}

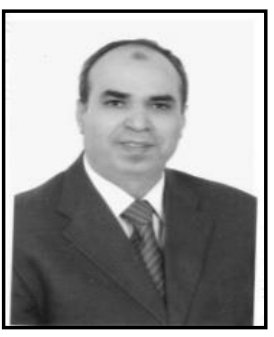

Mohammed Abo-Zahhad (SIEEEM'00) received his B.S.E.E. and M.S.E.E degrees in electrical engineering in 1979 and 1983 respectively, both from Assiut University, Egypt . In 1988, he received Ph. D. degree from the University of Kent at Canterbury, UK and Assiut University (channel system). His research interests include switched-capacitor, optical and digital filters, biomedical and genomic signal processing, speech processing, data compression, wavelet-transforms, genetic algorithms, immune algorithms, wireless sensor networks and electronic systems. He has published more than 106 papers in national and international journals and conferences in the above fields. Professor AboZahhad is currently a Professor of Electronics and Communi-cation Engineering, since Jan.1999. Also, he is the director of AU Management Information System (MIS) center and a vice-dean for graduated studies, Faculty of Engineering, Assiut University, since August 2006. He is a member of the European Society of Circuit Theory and Applications, 1998 and a senior IEEE member, 2000.

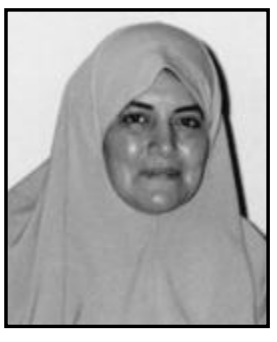

Sabah M. Ahmed received her B.S.E.E. and M.S.E.E degrees in electrical engineering in 1979 (excellent with honors) and 1983 respectively, both from Assiut University, Egypt . In 1992, she received Ph. D. degree from the Technical University of Budapest, Hungary. Her research interests include speech processing, biomedical and genomic signal processing, data compression, wavelet-transforms, genetic algorithms, and immune algorithms. She has published more than 56 papers in national and international journals and conferences in the above fields. Professor Sabah is currently a Professor of Electronics and Communication Engineering, since Feb. 2009. Also, she is the director of Faculty of Engineering ICDL center, Assiut University and the manager of Assiut University communication and information technology training center.

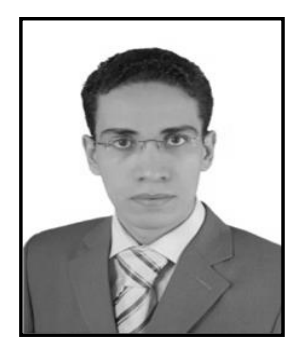

Nabil Sabor received his B.S.E.E and M.S.E.E. degrees in electrical engineering in 2006 (excellent with honors) and 2011 respectively, both from Assiut University, Egypt. His research interests include signal and image processing, data compression, biomedical signal processing, wavelet transform, digital filters, genetic algorithms, immune algorithms and wireless sensor networks. He has published more than 6 papers in national and international journals and conferences in the above fields. Mr. Nabil Sabor is currently an Assistant Lecturer in Department of Electronics and Communication Engineering, Assiut University, since May 2011. 


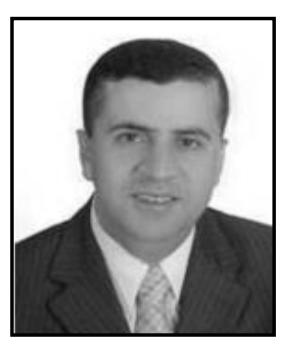

Ahmad Al-Ajlouni (SM'08) received his Ph.D. degree in electrical and computer engineering from Clarkson University, Potsdam, NY, in 1997. Dr. Al-Ajlouni is now a Professor of Computer and Communication Engineering and the dean of Hijjawi Faculty for Engineering Technology at Yarmouk University in Irbid, Jordan. His research interests are in the areas of computer networks, digital signal processing, active noise cancellation, artificial intelligence and engineering education. $\mathrm{He}$ is the author/co-author of more than 30 international published papers. 